\title{
Ultrafast evolution and transient phases of a prototype out-of-equilibrium Mott-Hubbard material
}

\author{
David Moreno-Mencía (i] ${ }^{1}$, Alberto Ramos-Álvarez ${ }^{1}$, Luciana Vidas ${ }^{1}$, Seyed M. Koohpayeh $^{2}$ \& Simon Wall (i] ${ }^{1}$
}

ARISING from G. Lantz et al. Nature Communications https://doi.org/10.1038/ncomms13917 (2017).

antz et $\mathrm{al}^{1}$. report a transient non-thermal phase in $\mathrm{V}_{2} \mathrm{O}_{3}$ following photoexcitation with femtosecond pulses of light at $800 \mathrm{~nm}$ arising from both the paramagnetic insulating (PI) and paramagnetic metallic (PM) phases of $\mathrm{V}_{2} \mathrm{O}_{3}$. The transient state is said to be stabilised by lattice distortion due to an overpopulation of the $\mathrm{a}_{1 \mathrm{~g}}$ electronic orbital. This is observed through a hardening of the $\mathrm{A}_{1 \mathrm{~g}}$ coherent phonon mode by $14 \%$ from the value observed in Raman measurements at equilibrium. We have repeated the optical measurements, performed on the paramagnetic metallic phase, on several different samples and found no evidence for light-induced phonon hardening. Raman and time-domain signals are found to be in good agreement. Instead, we find that the equilibrium $\mathrm{A}_{1 \mathrm{~g}}$ mode frequency is sample dependent, with values spanning $0.6 \mathrm{THz}$ in the three samples measured. Our results show that $\mathrm{V}_{2} \mathrm{O}_{3}$ does not undergo any anomalous photo-induced phase transition and that the excited state is most likely a thermal one.

The data shown in Fig. 1 comes from a single crystal of the PM phase of $\mathrm{V}_{2} \mathrm{O}_{3}$. The samples were grinded and polished using grinding disk papers and polishing cloths with diamond suspension down to $3 \mu \mathrm{m}$ to give a (001) surface normal. The room temperature lattice parameters of $\mathrm{a}=4.9535(5) \AA$ and $\mathrm{c}=$ 14.0043(4) Å were measured. A sharp first-order insulator-metal phase transition at $165 \mathrm{~K}$, often resulting in the crystal shattering, shows that our samples are of high quality and correspond to stoichiometric $\mathrm{V}_{2} \mathrm{O}_{3}$.

Experiments were carried out in an optical cryostat held at $200 \mathrm{~K}$. In order to reproduce the experimental conditions reported in Ref. ${ }^{1}$, the pump-probe measurements were performed in a cross-polarised geometry at near normal incidence, and the pump fluence was set to $8 \mathrm{~mJ} \mathrm{~cm}-2$. One significant difference in our measurement is that we use a broadband optical probe and frequency-resolve detection, which provide greater sensitivity to the phonon oscillation. Figure la shows the wavelength dependence of the transient optical response after correcting for the chirp of the white light probe. The response is similar to that observed by Ref. ${ }^{1}$ with a spike-like feature near $t=0$ and coherent oscillations over a slowly varying background. The response is similar at all wavelengths, but the relative strength of the peak and oscillation amplitude changes for different wavelengths. In order to obtain the phonon frequency, we averaged a 100-nm section of the wavelength data, centred at $600 \mathrm{~nm}$, to produce a single time trace. Figure $1 \mathrm{~b}$ shows a zoom of the transient, together with a time dependent fit to the transient data, which resulted in a central frequency of $7.41 \pm 0.01 \mathrm{THz}\left(247 \mathrm{~cm}^{-1}\right)$. In addition, we remove the background by differentiating the transient response, as performed by Ref. ${ }^{2}$, which acts as a complex low-pass filter for the slow dynamics. A fast Fourier transform (FFT) of the result was then calculated, after excluding the range close to the spike at $t=0$. The resulting FFT is shown in Fig. 1c. A Lorentzian fit to the FFT gives a central frequency of $7.51 \pm 0.01 \mathrm{THz}\left(250 \mathrm{~cm}^{-1}\right)$. The discrepancy in frequency obtained from the two methods of analysis of the same data is larger than the error reported by the fitting algorithm in either case. This shows that the main error in determining the mode frequency arises from how the background is processed. We determine the frequency of the mode to be $7.46 \pm 0.05 \mathrm{THz}$ by using the mean and standard deviation of the two fit results. This frequency is significantly slower than $8 \mathrm{THz}$, the response observed in Ref. ${ }^{1}$.

Raman measurements were also performed on the same sample at room temperature. Here, a small red shift can be expected due to the thermal induced softening of the mode, i.e., we expect it to be slightly lower in frequency than the data recorded at $200 \mathrm{~K}$. The data were recorded in an unpolarised backscattering geometry with a $785-\mathrm{nm}$ laser, and the results are also plotted in Fig. 1c. The Raman measurements are in good agreement with previous reports in the literature $^{3,4}$, again attesting to the good quality of our crystals. We used a multi-Lorentzian fit and linear background to extract the central frequency of the Raman mode and a value of $7.39 \pm 0.03 \mathrm{THz}$. Again, this value can change if the background function is also modified, and the true error will be larger than the value reported by the fit. However, even with this lower bound, the Raman and time-domain

\footnotetext{
${ }^{1}$ ICFO-Institut de Ciències Fotòniques, The Barcelona Institute of Science and Technology, 08860 Castelldefels, Barcelona, Spain. ${ }^{2}$ Department of Physics and Astronomy, Institute for Quantum Matter, Johns Hopkins University, Baltimore, MD 21218, USA. Correspondence and requests for materials should be addressed to S.W. (email: simon.wall@icfo.eu)
} 
a

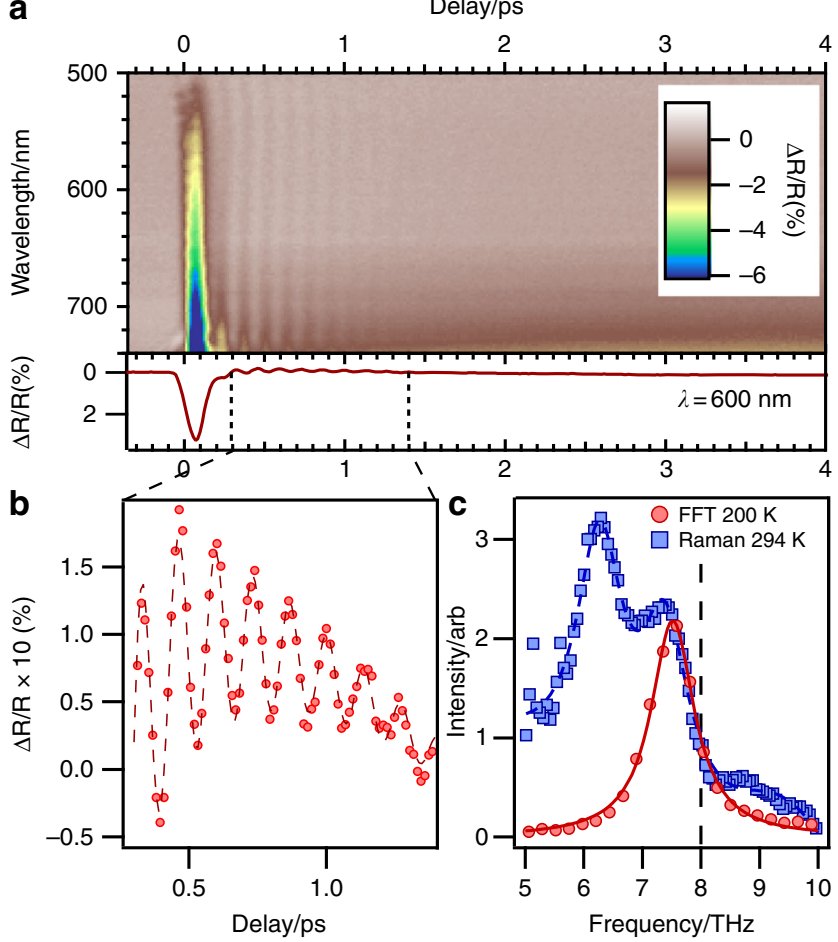

Fig. 1 Transient response of $\mathrm{V}_{2} \mathrm{O}_{3}$ to photoexcitation. a Time- and wavelength-resolved response of $\mathrm{V}_{2} \mathrm{O}_{3}$ after excitation with a $40 \mathrm{fs} 1.5 \mathrm{eV}$ pump pulse at $1 \mathrm{kHz}$ repetition rate, a pump fluence of $8 \mathrm{~mJ} \mathrm{~cm}^{-2}$ and a base temperature of $200 \mathrm{~K}$. The $2 \mathrm{D}$ data are averaged over a $100-\mathrm{nm}$-wide region, centred at $600 \mathrm{~nm}$ to extract a 1D time trace. $\mathbf{b}$ Zoom of the data to show the measured coherent phonon oscillations (circles) together with the fit (dashed line) with a 7.4 THz mode. c Fourier transform of the same data (blue circles) after differentiation, in order to remove the slow dynamics. A Lorentzian fit (blue line) gives a central frequency of 7.5 THz. The extracted frequency is in good agreement with the Raman data, taken at room temperature (blue line). The first peak $(\sim 6 \mathrm{THz})$ corresponds to the $\mathrm{E}_{\mathrm{g}}$ mode, which is not measured in our time-resolved measurement, and the second corresponds to the observed $A_{1 g}$ mode. The mode frequencies are in good agreement with Ref. ${ }^{3}$. The black dashed line is the blue-shifted frequency reported in Ref. ${ }^{1}$

frequencies obtained can be considered the same, to within the combined error bars. Therefore, we conclude that light does not induce a significant blue shift.

In order to investigate the discrepancy further, we performed a range of fluence-dependent measurements on different samples of stoichiometric $\mathrm{V}_{2} \mathrm{O}_{3}$ and at different fluences. Samples 1 and 2 were grown and polished by our collaboration at John Hopkins University using the methods described above. The data from other samples were acquired at facilities in our lab at ICFO. We show these data only for the purpose of showcasing range of frequencies that can be observed from sample to sample, but not for the purpose of reporting scientific values. Figure 2 plots the mode frequency as a function of power resulting from a Lorentzian fit to the Fourier transform of the background subtracted data. In sample 1, we find that the frequency of the response can change by $3 \%$ by moving to different spots in the same sample, which otherwise showed no obvious difference in the optical appearance (sample 1a vs sample $1 \mathrm{~b}$ in Fig. 2), indicating that residual strain from polishing may change the frequency. Agreement can also be found between different samples (samples 1a and 2). The largest discrepancy we have observed is $0.6 \mathrm{THz}$ (sample $1 \mathrm{~b}$ and

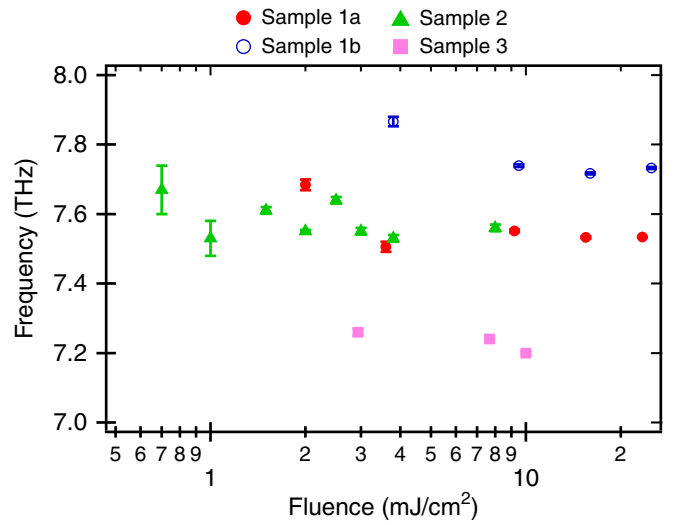

Fig. 2 Fluence dependence of the time-domain frequency for several samples of un-doped $\mathrm{V}_{2} \mathrm{O}_{3}$. All measurements were performed at room temperature. Samples $1 \mathrm{a}$ and $\mathrm{b}$ where measurements performed at two points on the same sample. A variation of $0.6 \mathrm{THz}(\sim 8 \%)$ is found over the samples measured. Error bars correspond to the error from the fit of the Lorentzian obtained from Fourier transforming the time-domain data and are an underestimate. Source data are provided as a Source Data file

sample 3). This shows the measured mode frequency is very sample dependent and depends strongly on sample preparation.

Lantz et al. ${ }^{1}$ argue that the blue shift is driven by the population in the $\mathrm{a}_{1 \mathrm{~g}}$ electronic level. Therefore, one would expect the magnitude of the shift to increase or decrease with increasing or decreasing fluence as more or fewer electrons are excited into that orbital. However, we only observe a small red shift with fluence, despite changing the fluence by almost two orders of magnitude. Over this range, the phonon amplitude and reflectivity change continue to increase, showing that the $\mathrm{a}_{1 \mathrm{~g}}$ orbital population has not saturated.

In conclusions, we find no evidence for a transient nonthermal phase in $\mathrm{V}_{2} \mathrm{O}_{3}$ that is stabled by a lattice distortion and photo-induced hardening of the $\mathrm{A}_{1 \mathrm{~g}}$ phonon mode as claimed in Ref. ${ }^{1}$. Good agreement between Raman and time-domain signals can be found when the measurements are performed on the same sample. There is no light-induced hardening and we do not find any evidence for control of the lattice structure by selective excitation of the $\mathrm{a}_{1 \mathrm{~g}}$ orbital in this compound. Our results show that there can be a large variation in $\mathrm{A}_{1 \mathrm{~g}}$ phonon mode frequency both within and between samples, which needs to be taken into account when comparing measurements with literature values.

The data that support the findings of this study are available from the corresponding author upon reasonable request.

Received: 13 July 2018 Accepted: 31 July 2019

Published online: 06 September 2019

\section{References}

1. Lantz, G. et al. Ultrafast evolution and transient phases of a prototype out-ofequilibrium Mott-Hubbard material. Nat. Commun. 8, 13917 (2017).

2. Misochko, O. et al. Optical study of the Mott transition in $\mathrm{V}_{2} \mathrm{O}_{3}$ : comparison of time- and frequency-domain results. Phys. Rev. B 58, 12789-12794 (1998).

3. Kuroda, N. \& Fan, H. Y. Raman scattering and phase transition of $\mathrm{V}_{2} \mathrm{O}_{3}$. Phys Rev. B 16, 5003 (1977).

4. Tatsuyama, C. \& Fan, H. Raman scattering and phase transitions in $\mathrm{V}_{2} \mathrm{O}_{3}$ and $\left(\mathrm{V}_{1-\mathrm{x}} \mathrm{Cr}_{\mathrm{x}}\right)_{2} \mathrm{O}_{3}$. Phys. Rev. B 21, 2977-2983 (1980).

\section{Acknowledgements}

Work at ICFO was supported by Spanish MINECO (Severo Ochoa grant SEV-20150522), Ramón y Cajal programme RYC-2013-14838, Fondo Europeo de Desarrollo Regional FIS2015-67898-P (MINECO/FEDER), Fundació Privada Cellex, CERCA 
Programme/Generalitat de Catalunya and from the European Research Council (ERC) under the European Union's Horizon 2020 research and innovation programme (grant agreement no. 758461). The work at the Institute for Quantum Matter, an Energy Frontier Research Center, was supported by the U.S. Department of Energy, Office of Science, Basic Energy Sciences under Award Number DE-SC0019331.

\section{Author contributions}

D.M.M., A.R.A. and L.V. built the time-domain experimental setup, measured the static Raman data and analysed the data under the supervision of S.W. S.M.K. grew and characterised the static properties of the $\mathrm{V}_{2} \mathrm{O}_{3}$ single crystals studied. S.W. wrote the paper and coordinated the project.

\section{Additional information}

Supplementary Information accompanies this paper at https://doi.org/10.1038/s41467019-11743-3.

Competing interests: The authors declare no competing interests.

Reprints and permission information is available online at http://npg.nature.com/ reprintsandpermissions/
Peer review information: Nature Communications thanks Donald Walko and other anonymous reviewer(s) for their contribution to the peer review of this work.

Publisher's note: Springer Nature remains neutral with regard to jurisdictional claims in published maps and institutional affiliations.

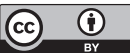

Open Access This article is licensed under a Creative Commons Attribution 4.0 International License, which permits use, sharing, adaptation, distribution and reproduction in any medium or format, as long as you give appropriate credit to the original author(s) and the source, provide a link to the Creative Commons license, and indicate if changes were made. The images or other third party material in this article are included in the article's Creative Commons license, unless indicated otherwise in a credit line to the material. If material is not included in the article's Creative Commons license and your intended use is not permitted by statutory regulation or exceeds the permitted use, you will need to obtain permission directly from the copyright holder. To view a copy of this license, visit http://creativecommons.org/ licenses/by/4.0/.

(C) The Author(s) 2019 